\title{
Improving the Accuracy of Low-Rigid Shaft Lathing in Self-Centering Steady
}

\author{
Oleg Drachev ${ }^{1, *}$, Boris Gorshkov ${ }^{2}$, Victor Tisenko ${ }^{3}$, and Natalia Samokhina ${ }^{2}$ \\ ${ }^{1}$ Togliatti State University, Togliatti, Russia \\ ${ }^{2}$ Volga Region State University of Service, Togliatti, Russia \\ ${ }^{3}$ Peter the Great St.Petersburg Polytechnic University, Russia
}

\begin{abstract}
Device for axis-stabilizing of low-rigid work pieces has been presented in the article. The method of calculation of dynamic parameters of the "work pieces-supports" subsystem is discussed. The influence on dynamic of work pieces by different variants of fixation is explored.
\end{abstract}

\section{Introduction}

Low-rigid shaft workpieces are usually clamped for turning in a self-centering chuck with a rear center tightening and using an adjustable steady rest. Due to the possible misalignment of the axes of the process system base elements, the workpiece axis can be bent when it is force-locked in the axial direction by the rear center. When machining, shaft deflection also increases due to thermal and inertial disturbances in force in the process system. Accordingly, the problem of the shaft axis stabilizing requires a further research to be solved. High demands are placed on deviations in the shape and location of the base surfaces for the rests, in which case either alignment of the workpiece position or turning of a preliminary base neck groove is required. To reduce the influence of the workpiece imbalance on the cutting process, it is necessary to decrease the machining modes.

When machining a nonrigid workpiece, it is necessary to reduce its bend and make it uniform along the length of the workpiece under the cutting forces action, as well as to reduce the imbalance of the rotating workpiece, and eliminate the influence of the force lock by the rear center.

The surface shaping during turning/boring is determined by the process system elements vibrations. At the same time, the regular macro and micro profile of the surface depends on polyharmonic oscillations with a stochastic component, which are a function of the spatial relationships and rigidity of the process system elements. If they are stable and do not vary during the machining, the surface profile is regular. The harmonic components of a workpiece profile are set by the vibration modes. Moreover, each of the profile harmonics (or a group of such) depends on a separate oscillation component determined by a certain element of the process system. During the low-rigid workpiece processing, the predominant influence on the machining accuracy is exercised by the workpiece supports subsystem offsets $[1 \div 10]$.

\footnotetext{
*Corresponding author: kaf_ekis@tolgas.ru
} 


\section{Relevance}

The challenge of the improving nonrigid shaft machining accuracy, shape and geometric dimension stability is one of the most pressing. To meet this challenge, the following problems were solved: development of a self-centering hydraulic steady rest design; pilot testing of the steady rest design under various machining conditions to stabilize a machined workpiece axis; theoretical justification of the use of various support combinations; analysis of the installation diagrams of nonrigid shafts in a turning operation in various modes..

In the process of nonrigid workpiece (NRW) machine turning, deep hole drilling, vibration drilling, etc., self-centering hydraulic rests (SCR) are widely used, which make it possible to stabilize the axis of a low-rigid workpiece (LRW) during processing [1, 4, 6, 9 ]. The design of the SCR is shown in Fig. 1.

The steady rests are placed along the workpiece length from the condition of equal rigidity as a function of the ratio of the workpiece lengths and diameters $(1 / \mathrm{d}<5)$ or the steady rests are installed in the zones of distribution antinodes of the workpiece higher basic vibration modes. Self-centering hydraulic steady rests are installed in the nodes of bending vibrations, and work as equally rigid supports. At that, the pressure supplied to the SCR provides the workpiece clamping, while on the controlled SCRs which are installed relative to the surface unprocessed in a given passage and operate in a damper mode, a pressure is created that provides suppression (damping) of lateral vibrations.

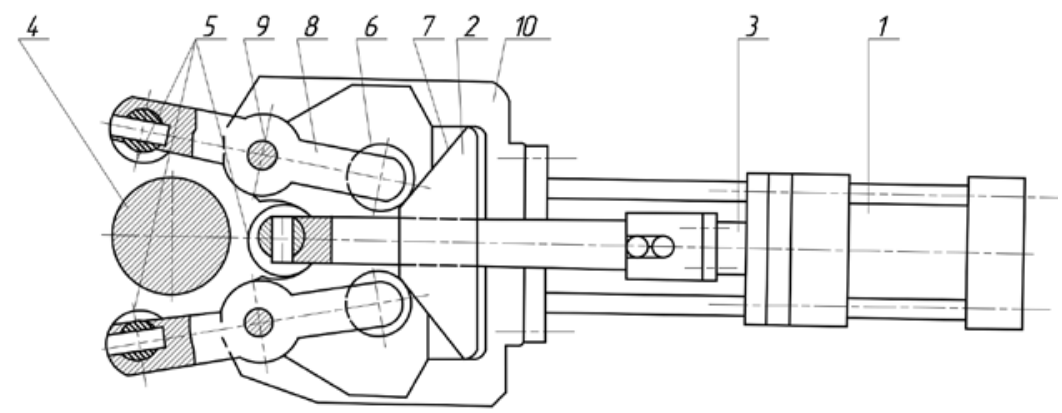

Fig. 1. Self-centering steady rest.

Whereas, in the course of the drill movement along the workpiece, the damping SCR which operate in the damping mode are switched to the equal support mode using the switcher as the function of the path traveled by the boring bar recorded by the sensor. In the initial state, before the cutting process, all hydraulic steady rests work in the mode of dampers, except for those working in the equal rigidity mode, which are installed at the head and tail stock. This condition is fulfilled in order to center the workpiece axis relative to the machine technical axis, and the workpiece supporting surfaces in the places where the extreme rests are installed are pre-lathed. The conventional method of a workpiece clamping leads to its axis curvature and to the occurrence of residual stresses in the material, which is enhanced by plastic deformation during the material machining. This allows the workpiece to be based on the workpiece surface generatrix coinciding with the measuring base surface, which leads to enhancing the machining accuracy and the exclusion of processing heredity from the clamping conditions.

During cutting, the SCR actuator works as follows: when the working medium (oil) is fed to the hydraulic cylinder of the drive 1, the flat copier 2 connected to the piston rod 3 of the drive moves to the workpiece 4, and the working rollers 5 (which can be doubled and have two degrees of freedom of rotation: relative to its own axis and to the axis of rotation) clamp or unclamp the workpiece by contacting the tracking rollers 6 on the flat copier 
working surface 7 . The copier working surface is implemented along a curve close to the radius and is calculated depending on the range of the workpiece diameters. One pair of the working rollers is located on the flat copier rod, two other pairs of rollers are fixed on levers 8 . The levers at the ends of which working rollers 5 are located are rotated relative to the axis of rotation 9 . The latter is fixed in the steady rest case 10 . The workpiece 4 is clamped by rollers according to a rigid program from a copier. The force closure of the rollers is implemented with a force proportional to the force developed by the drive. In order to unload the rollers and levers, as well as to increase the dynamic characteristics of the process system, the longitudinal axis of the steady rest body, together with the drive, is rotated relative to the horizontal plane by an angle a which is calculated as a function of the direction of the cutting force radial component depending on the geometric parameters of the cutting tools used: boring bar cutters, external turning cutters, drills. Experimental investigation of the low-rigid shaft axis behavior during lathing was carried out on a laboratory plant, comprising: 1A616 machine, workpiece length $450 \mathrm{~mm}$, outer diameter 50 $\mathrm{mm}$, inner diameter $40 \mathrm{~mm}$.

In fig. 2. shows the installation of self-centering steady rest installation on a lathe when machining a hole in a nonrigid shaft and SCR during machining.

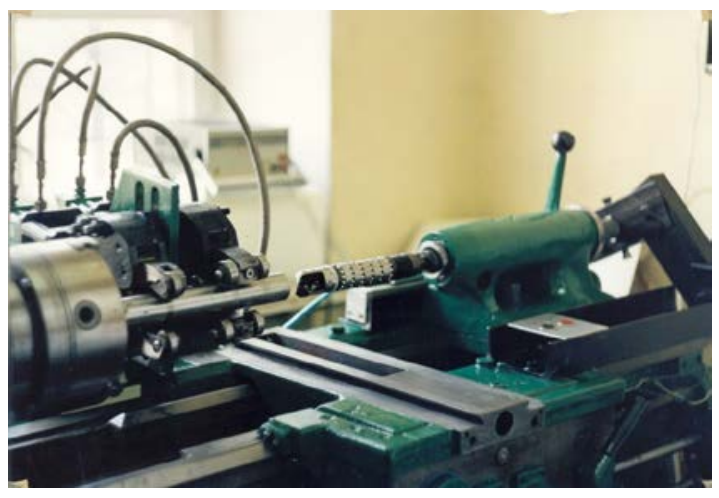

Fig. 2 Self-centering steady rest installation on a lathe when machining a hole in a nonrigid shaft

Analysis of the experimental dependences has shown that during cutting a workpiece axis shape changes with the increase in the rotation frequency: at a low rotation frequency, the curve shape is close to the static one; with an increase in the rotation frequency, the axis shape is deforming. During cutting, the low-rigid axis shape is close to that of the workpiece at idle, and the value of displacement of the SM axis depends on the nature of the cutting process and increases 23 times with the occurrence of self-oscillations. With a decrease of the workpiece bending stiffness, not only does the displacement of the axis increase, but also its shape changes; its difference from the static deflection curve increases. The stabilization system of the LRW axis in the described experiments included two SCRs. Turning using the system was carried out applying the same modes and workpieces as without the system. The results of the experiments showed that the greatest displacement of the workpiece axis during turning with the system decreased by $4 \ldots 10$ times. Workpieces $\mathrm{L}$ $=450 \mathrm{~mm}$, hole diameter $\mathrm{d}=42 \mathrm{~mm}$, were processed with a two-edge boring head without and with the system under the following cutting conditions: $n=710 \mathrm{rpm}, \mathrm{S}=0.27-0.5$ $\mathrm{mm} / \mathrm{rev}$; without the system - in two passes with the cut depth of $\mathrm{t} 1=0.7 \mathrm{~mm}$ and $\mathrm{t} 2=0.3$ $\mathrm{mm}$; with the system - in one pass $\mathrm{t}=1 \mathrm{~mm}$.

In the initial state, before the cutting process, all SCR operate in the mode of dampers, except for those installed at the headstock and tailstock and working in the mode of rigid supports. This condition is necessary for the workpiece axis centering relative to the 
technical axis of the machine. The workpiece supporting surfaces in the places where the steady rests are installed are preliminarily rolled to correct the base surface shape.

When applying the system, the biggest deviation from cylindricality decreased by $3 \ldots 5$ times, but the main thing is that the profilograms show a significant approximation of the workpiece shape to the cylindrical on, which cannot be said when measuring diameters in various sections. A TAYLOR-HOBSON device was used to construct circular diagrams of workpiece cross-sections to be utilized as reference surfaces. The analysis of the circular diagrams showed that the base surface deviation from roundness decreases by $1.3 \ldots 2$ times depending on the physical and mechanical properties of the material, its initial profile, and the working pressure. In the course of the experiment, the workpiece axis displacements were recorded in three sections: at idle speed, then in the process of cutting. From the oscillation analysis it follows that the workpiece imbalance per revolution determines the low-frequency component (time of one turn $\mathrm{T}=0.0615 \mathrm{~s}$ ) on which high-frequency oscillations are superimposed, and in the case of applying the SCR, their frequency increases and the amplitude decreases. Higher harmonics in the frequency spectrum of the spindle system are determined by the error in the manufacture of guide supports (in this case, spindle ball bearings and SCR). The workpiece is rigidly fixed and determines the low-frequency vibrations.

The frequency spectrum shifts to the high-frequency region in proportion to the increase in the rotation speed. The natural frequency of the SCR support elements is higher (the design dimensions of the ball bearings of the spindle and the SCR differ by an order of magnitude), and the amplitude of the disturbances turns out to be lower. When a workpiece rotates, the SCR dampens disturbances from the spindle bearings in the frequency range of 100 to $500 \mathrm{~Hz}$ (act as dampers of workpiece high-frequency displacements). This sample was also characterized by the minimum values of a hole run or diameter enlargement, by small amplitude of transverse vibrations, and the presence of intense longitudinal torsional vibrations.

The task of operating the cutting dynamics is reduced either to the control of the disturbances frequency in the function of changing the system natural frequencies through altering the processing mode, or to the control of the SCR working medium pressure in such a way that, for new combinations of rigid and damping supports, the subsystem natural frequency would change minimally, thereby, stabilizing the reduces rigidity and damping coefficients of the "workpiece-support" subsystem.

To substantiate the use of various combinations of supports, an analysis was made of the schemes for installing nonrigid shafts for a turning operation in stationary steady rests operating in various modes.

\section{Theoretical Substantiation of the Nonrigid Shaft Machining Dynamics Control}

To calculate a workpiece axis shape during turning, the method of initial parameters $[2,3]$ was applied, wherein for each segment transition matrices were compiled, which matrices then were sequentially multiplied. When passing to an intermediate support, an intermediate support matrix is to be compiled and multiplied by the previous result, then, matrices for the next segments and sections are to be compiled. Thus, a matrix for a multisupport complex shaft is obtained.

Fig. 3 shows a design diagram of a workpiece installed within five steady rests, where it is presented as a set of segments of weightless rods and concentrated masses, and the steady rests are replaced by supports elastic to transverse displacements. 


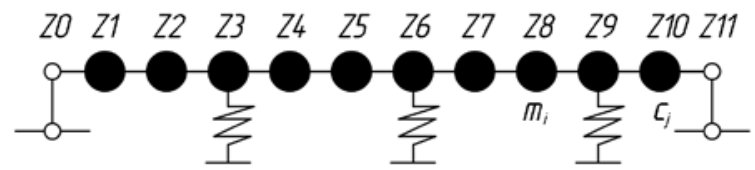

Fig. 3. Design diagram for determining a workpiece axis shape and natural frequencies

A transfer matrix for a design diagram is obtained by multiplying matrices of its elements.

$$
P=G_{n} M_{n} Q_{n} G_{n-1} G_{n-1, n-2} \ldots M_{k} Q_{k} G_{k-1} \ldots M_{0} Q_{0} \quad,
$$

where $G_{i}, M_{i}, Q_{i}$ are transition matrices for a weightless segment of the rod, a concentrated mass, and a support elastic to transverse displacements; $i$ is a segment number, $i=0 . . n ; n$ is the number of segments.

The main matrix equation connecting the boundary sections taken in the form of hinged supports:

or in the expanded form:

$$
Y_{n}=P Y_{0}
$$

$$
\left(\begin{array}{c}
Y_{n} \\
\varphi_{n} l \\
\frac{M_{n} l^{2}}{E J} \\
\frac{Q_{n} l^{3}}{E J}
\end{array}\right)=\left(\begin{array}{llll}
a_{11} & a_{12} & a_{13} & a_{14} \\
a_{21} & a_{22} & a_{23} & a_{24} \\
a_{31} & a_{32} & a_{33} & a_{34} \\
a_{41} & a_{42} & a_{43} & a_{44}
\end{array}\right) \cdot\left(\begin{array}{c}
Y_{0} \\
\varphi_{0} l \\
\frac{M_{0} l^{2}}{E J} \\
\frac{Q_{0} l^{3}}{E J}
\end{array}\right),
$$

where $E J$ is the bending stiffness of a certain segment of a shaft; $l$ is the shaft length; $y_{i}, Q_{i}$, and Mi are transverse displacement, angle of rotation, shear force, and bending moment in the i section; $\mathrm{a}_{\mathrm{ik}}$ are the transfer matrix coefficients.

The concentrated mass matrix Mi in the i section has the form:

$$
M=\left(\begin{array}{cccc}
1 & 0 & 0 & 0 \\
0 & 1 & 0 & 0 \\
0 & 0 & 1 & 0 \\
v_{i} & 0 & 0 & 1
\end{array}\right),
$$

where $v_{i}=\frac{m_{i} \omega^{2} l^{3}}{E J} m_{i}$ is the mass reduced to the section $i$; $\omega$ is natural circular

vibration frequency.

The stiffness transition matrix for a weightless shaft segment of $L_{i}$ length and $\mathrm{EJ}_{\mathrm{i}}$ stiffness looks like as follows:

$$
G_{i}=\left(\begin{array}{cccc}
1 & h_{i} & \frac{h_{i}^{2}}{2 \cdot u_{i}} & \frac{h_{i}^{3}}{6 \cdot u_{i}} \\
0 & 1 & \frac{h_{i}}{u_{i}} & \frac{h_{i}^{2}}{2 \cdot u_{i}} \\
0 & 0 & 1 & h_{i} \\
0 & 0 & 0 & 1
\end{array}\right),
$$

where $h_{i}=l_{i} / l$ and $l_{i}=i$ segment length, $L$ is the workpiece length; $u_{i}=E J_{i} / E J$, where $\mathrm{EJ}_{\mathrm{i}}$ is the i segment bending stiffness. The shaft stiffness in this segment is assumed to be constant.

The cutting force acting on the shaft may be taken as changing under the sine law with a frequency corresponding to the workpiece rotation frequency or a multiple thereof. The disturbance periodicity is due to the variable allowance, as also to the workpiece 
imbalance. For the bar weightless segment loaded with a concentrated harmonic (cutting) force the relationship between the parameters in sections $i$ and $i-1$ is as follows:

where

$$
Y_{i}=G_{i} Y_{i-1}+\psi_{i}
$$

$$
\psi=\left(\begin{array}{c}
0 \\
0 \\
0 \\
\frac{P_{l} l^{3}}{E J}
\end{array}\right)
$$

where $\mathrm{Pi}$, is the amplitude of the harmonic force changing with the frequency $\Omega$.

The transition matrix of a rod weightless section with a support elastic relative to transverse displacements has the following form:

$$
Q_{i}=\left(\begin{array}{cccc}
1 & h_{i} & \frac{h_{i}^{2}}{2 \cdot u_{i}} & \frac{h_{i}^{3}}{6 \cdot u_{i}} \\
0 & 1 & \frac{h_{i}}{u_{i}} & \frac{h_{i}^{2}}{2 \cdot u_{i}} \\
0 & 0 & 1 & h_{i} \\
0 & 0 & 0 & 1-\frac{e_{i} \cdot u_{i}^{3}}{6 \cdot u_{i}}
\end{array}\right),
$$

where $e_{i}=\frac{c_{i} l^{3}}{E J} ; \mathrm{c}_{\mathrm{i}}$, is the support rigidity coefficient.

Four components of the $\mathrm{Y}_{0}$ and $\mathrm{Y}_{\mathrm{n}}$ matrices are defined, two of which for the hinged supports at the shaft ends are equal to zero. The column matrices of parameters in the zero and in the i section are:

$$
Y_{0}=\left(\begin{array}{c}
0 \\
\varphi_{0} l \\
0 \\
\frac{Q_{0} l^{3}}{E J}
\end{array}\right) \quad ; Y_{n}=\left(\begin{array}{c}
0 \\
\varphi_{n} l \\
0 \\
\frac{Q_{n} l^{3}}{E J}
\end{array}\right)
$$

From the first and third lines of expression (3) we find:

$$
\left.\begin{array}{l}
0=a_{12} \varphi_{0} \cdot l+Q_{0} \cdot \frac{l^{3}}{E J} \\
0=a_{32} \varphi_{0} \cdot l+Q_{0} \cdot \frac{l^{3}}{E J}
\end{array}\right\}
$$

In the transition matrices, when calculating forced vibrations, the shaft natural frequency $\omega$ is replaced by the disturbance frequency $\Omega$. To calculate forced vibrations, the obtained system (10) is solved, and deflections and angles of rotation at the shaft right end are found. Using the known parameters $\varphi_{0}$ and $\mathrm{Q}_{0}$ and transfer matrices for multiplying them, the parameters in any section are found. Further, other frequency values are selected and the corresponding deflections are found by repeating the same calculation scheme. All coefficients and constants are found from the experimental data. 


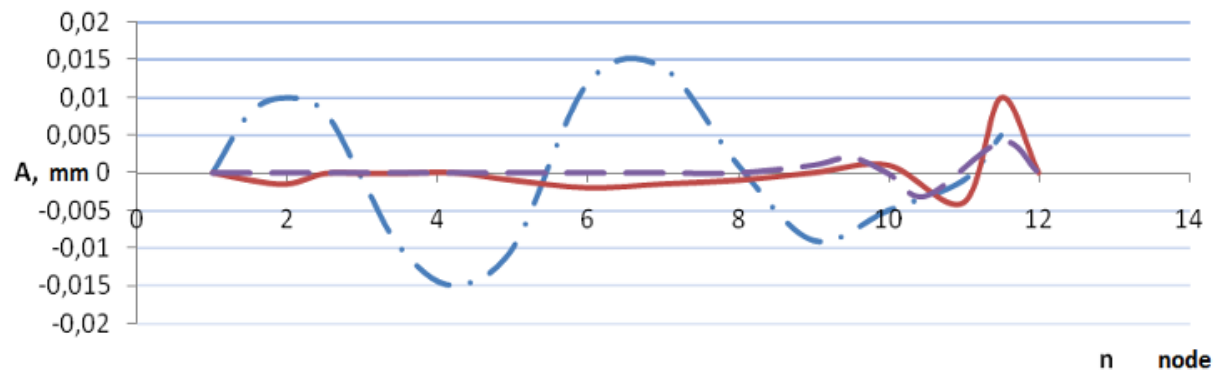

Fig. 4. A workpiece axis shape under various conditions of clamping, forcing frequency, and cutting zone location at the $\mathrm{n} 10$ node: the support at the $\mathrm{n} 9$ node is rigid with rigidity coefficient of $\mathrm{C}=$ $2 \cdot 10^{6} \mathrm{~N} / \mathrm{m}$, the rest are damping, $\mathrm{C}=0.5 \cdot 10^{6} \mathrm{~N} / \mathrm{m}, \Omega=314 \mathrm{rad} / \mathrm{s}$; all supports are rigid, $\mathrm{C}=$ $2 \cdot 10^{6} \mathrm{~N} / \mathrm{m}$, disturbance frequency $\Omega$ is from 31.4 to $62.8 \mathrm{rad} / \mathrm{s}$; all supports are damping, $\mathrm{C}=$ $0.5 \cdot 10^{6} \mathrm{~N} / \mathrm{m}, \Omega=314 \mathrm{rad} / \mathrm{s}$.

The results of calculations following this algorithm are given in Figs. 4 and 5. Especially noteworthy is the variation in the amplitude of the workpiece displacement in the cutting zone depending on the change in the nature of the supports, forcing frequency, and the cutting zone location. All these technical factors also lead to a change in the shape of a low-rigidity workpiece axis, which, as mentioned above, additionally affects the cutting zone due to the workpiece imbalance in the segments remote from this zone. The kinetic energy is transferred to the cutting zone and also contributes to varying the parameters of the amplitude of the workpiece and cutter relative oscillations.

Analysis of the forms of forced vibrations of a 5-support nonrigid long shaft (rigidity is $10 \ldots 100$ times less than that of the supports) loaded in the cutting zone with an alternating force and a harmonic load, depending on the vibration frequency showed that within the frequency range from 0 to $100 \mathrm{~Hz}$ the first through the fifth of its own form can be excited. Bringing the supports closer to each other makes it possible to reduce errors caused by the "workpiece-support" subsystem elastic deformations to values commensurate with other machining errors so that the workpiece elastic deformations do not dominate the total error.

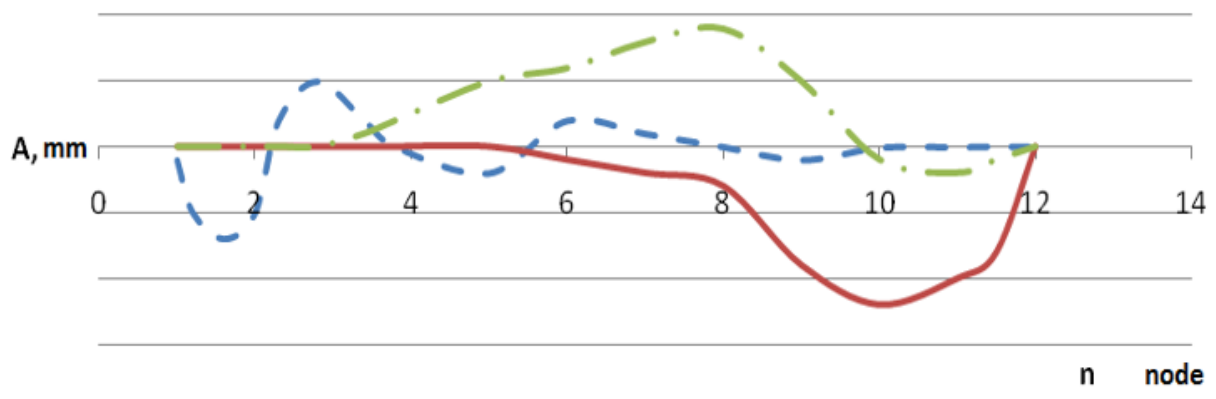

Fig. 5. A workpiece axis shape under various conditions of clamping, forcing frequency, and cutting zone location at the $\mathrm{n} 8$ node: the supports at the $\mathrm{n} 6$ and $\mathrm{n} 9$ nodes are rigid, $\mathrm{C}=2 \cdot 10^{6} \mathrm{~N} / \mathrm{m}$, the rest are damping, $C=0.5 \cdot 10^{6} \mathrm{~N} / \mathrm{m}, \Omega=314 \mathrm{rad} / \mathrm{s}$; all supports are damping, $C=0.5 \cdot 10^{6} \mathrm{~N} / \mathrm{m}, \Omega=31.4$ $\mathrm{rad} / \mathrm{s}$; all supports are damping, $\mathrm{C}=0.5 \cdot 10^{6} \mathrm{~N} / \mathrm{m}, \Omega=628 \mathrm{rad} / \mathrm{s}$.

The experimental study of a low-rigid workpiece axis behavior in the process of turning and at idle was conducted on an industrial plant based on an RV106 (PB106) screw-cutting 
lathe. A shaft workpiece of $30 \mathrm{~mm}$ in diameter and $3000 \mathrm{~mm}$ in length made of $30 \mathrm{KhN} 3 \mathrm{~A}$ material was installed in five steady rests. The extreme rests worked as rigid supports and set the workpiece axis of rotation. The central steady rests were switching from the rigid support mode with the static stiffness coefficient $\mathrm{K}=20 \cdot 10^{6} \mathrm{~N} / \mathrm{m}$ to the damping support mode with the stiffness coefficient $K=20 \cdot 10^{6} \mathrm{~N} / \mathrm{m}$.

A signal from a BENTLY-NEVADA-type eddy current sensor was sent through an amplifier to a PC. In the course of the experiment, the displacements of the workpiece axis were recorded in its cross-sections at idle speed, and then during cutting.

The experimental results demonstrated that the largest displacement of the shaft axis during turning with the system decreased by $4 \div 10$ times. The workpiece axis shape during cutting is close to that at idle. When measuring at idle, changes of the workpiece oscillation amplitude displacements were detected in the same section with parallel changing the variants of combinations of supports of different type (from 0.03 to $0.05 \mathrm{~mm}$ ). As calculations had shown, a change in the cutting zone position for various combinations of supports leads to different values of a workpiece longitudinal displacements.

As the spindle speed $\mathrm{n}$ grows from 120 to $400 \mathrm{rpm}$, a workpiece displacement amplitude in SCR increases from 0.01 to $0.021 \mathrm{~mm}$. A workpiece imbalance through a revolution determines a low-frequency component of vibrations (one revolution time $\mathrm{T}=0.6-1.15$ sec.) on which high-frequency oscillations are superimposed. The disturbing force higher harmonics arise due to a workpiece variable dimensional allowance because of the form errors (ovality, faceting). The higher harmonics in the frequency spectrum of the spindle system are determined by manufacture errors of guide supports (in this case, spindle and SCR ball bearings). When rotating a workpiece, SCR suppress disturbances from spindle bearers in the frequency range of 100 to $500 \mathrm{~Hz}$. Without an SCR, these frequencies are close to the higher harmonics of a workpiece itself and increase due to this.

\section{Conclusion}

Theoretical calculations have shown that a workpiece displacement is less for the cases of all rigid steady rests and two rigid steady rests between which the cutting zone is located, all the other being damping supports. The experiment demonstrated that in the instance of machining workpieces with a sizeable initial curvature $(1.5 \mathrm{~mm}$ per $1 \mathrm{~m}$ of length), it is more expedient to use all the steady rests in the damping mode. During clamping a workpiece, rigid steady rests align the workpiece axis due to its elastic deformation with the machine technical axis. After machining, when the workpiece is unclamped, its elastic deformation leads to a significant error. In the case of damping steady rests, the elastic deformation of a workpiece decreases. For the machining of workpieces with a smaller warpage value, the option with the rigid-to-damping switchable supports in more preferable.

The schemes of clamping a nonrigid workpiece in the device for stabilizing the workpiece axis by the method of initial parameters were analyzed. The application of various combinations of supports with the choice of a rational location for the rigid and (or) damping steady rests was substantiated to increase the stationarity of the machining process and to improve the quality of shaping a machined surface. The use of a system for stabilizing axes of low-rigid shaft-type workpieces during one-pass machining operations under intensive cutting conditions may provide an error of at most 12 microns with a roughness not exceeding $\mathrm{Ra} 6.3$ microns due to the low-rigid workpiece dynamics control. The developed installation together with the technology of its use was introduced at OAO Azotremmash for rotor shafts machining. 
By using in the SCR rollers needle bearings instead of ball bearings it is practically possible to achieve the LRW displacement in the form of a pure sinusoid, which will increase the cutting process stationarity.

The design of a workpiece subsystem on the basis of self-centering hydraulic steady rests has been developed to provide an increase in the transversal rigidity of the workpiece during machining by $9.4 \div 12$ times as a function of the working pressure in the SCR hydraulic drive.

\section{References}

1. O. Draczow, W. Taranenko. Technologia modulowa wytwarzanm osiowosymetiycznych waiow dlugowymiarowych. ZESZYTY NAUKOWE Politechniki Rzeszowskiej nr 230 MECHANIKA, z. 67 -Modulowe Technologje i Konstrukcje w Budowe Maszyn, Rzeszow, pp. 47-50 (2006).

2. M. Orlikov. Machine dynamics, - K.: Vysshaya shkola.-272 p., (1989)

3. V. Ivanovich. Transition matrices in elastic system dynamics. - M.: Mashinostroyenie (1969)

4. O. Drachev, A.Kravtsov. Automatic control of the process of low-stiff parts turning. Togliatti: VSTU, ZAO «ONIKS». (2012)

5. E.Artamonov, A.Tveryakov, A.Shtin. Definition of the temperature of the ultimate operational capability of instrumental hard alloys. Obrabotka metallov (tekhnologia, oborudovanie, instrumenty) - Metals processing (Technology, Equipment, Tools. (2018).

6. A.Bobrowski, O.Drachev, B.GorshkovM. Improving the operational accuracy of shafts by volumetric plastic deformation. Available online. (2020).

7. V. Zakovorotniy, D.Voloshin, S.Rodriges, M. Flek. Method of modeling elastic deformations of cylindrical end mills in the process of milling. Investigation of machines and tools for machining complex and precise surfaces. Saratov: SSTU. (2003).

8. A. Vladimirov, A.Sergiev. Influence of vibrational amplitude on component functions of the complex vibrational effect of low-frequency vibrations. Izvestiya VolgGTU The Bulletin of VolgGTU, 7, pp. 13-17. (2018).

9. A.Bobrovsky, O. Drachev, A. Kravtsov. Conference Series: Materials Science and Engineering. Collection of materials of the International Conference on Modern Trends in Manufacturing Technologies and Equipment: Mechanical Engineering and Materials Science (ICMTMTE 2019). Sevastopol State University, National University of Science and Technology «MISiS», Polzunov Altai State Technical University, Crimean Federal University, INLINK Ltd. and International Union of Machine Builders. Page 044088. (2020)

10. F. Chemavskiy. Modern trends in the construction of precast cutters with nonreversible plates, Review. Moscow: NIIMash. (1983) 